\section{Consideraciones generales del uso de anestesia intravenosa total}

General considerations for the use of total intravenous anesthesia

Joselyn Anabell Minda Reyes

Médico general. Centro Médico

Medilink,josyanabell@hotmail.com,

https://orcid.org/0000-0002-9476-

3222, Guayaquil, Ecuador

Dayana Lissette Miranda Espín

Médico general. Centro de Salud

Socio vivienda 1,

dra.dayimiranda@gmail.com,

https://orcid.org/0000-0001-5073-

3892, Guayaquil, Ecuador

Elsa Rebeca Pilamunga Valla

Médico general. Hospital General

Martín Icaza,

md.pilamungarebeca@gmail.com,

https://orcid.org/0000-0002-2524-

2554, Guayaquil, Ecuador

Jazmín Katiusca Anchundia

Salvatierra

Médico general. Coordinación

provincial de prestaciones del seguro

de salud del Guayas,

katigatiu@yahoo.com;

https://orcid.org/0000-0002-7462-

8832, Guayaquil, Ecuador

http://www.jah-

journal.com/index.php/jah

Journal of American health

Julio - Diciembre vol. 3. Num. 2 - 2020

Esta obra está bajo una Licencia

Creative Commons

Atribución-NoComercial-

CompartirIgual 4.0 Internacional.

RECIBIDO: 3 DE FEBRERO 2019

ACEPTADO: 10 DE AGOSTO 2019

PUBLICADO: 2 DE JULIO 2020

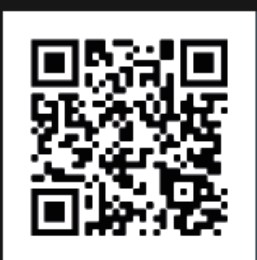

Scan this QR

code with your

smart phone or

mobile device to

read more papers

\section{RESUMEN}

Los avances recientes de salud, especialmente en el campo de la cirugía requiere un mejor control anestésico intraoperatorio y un mínimo de efectos adversos en el post operatorio, para esto actualmente se dispone nuevos agentes inductores como el propofol, se logra realizar el mantenimiento anestésico sin la necesidad de un agente inhalatorio. El objetivo principal de esta investigación fue presentar actualizaciones recientes en la literatura sobre anestesia intravenosa total, enfocada en sus efectos farmacológicos y sus principales indicaciones. Se realizó una revisión de la literatura y un análisis de publicaciones sobre anestesia intravenosa total en los últimos 10 años, empleando bibliotecas digitales de Pubmed y Scielo, tanto de artículos en español e inglés. La anestesia total intravenosa es la solución predeterminada para un paciente con riesgo de hipertermia maligna que requiere anestesia general, además de disminuir el riesgo de efectos secundarios de la anestesia general, como náuseas y vómitos postoperatorios y evitan la contaminación del aire ambiental con los agentes inhalantes.

PALABRAS CLAVE: anestesia intravenosa, propofol, remifentanilo, farmacocinética.

\section{ABSTRACT}

Recent health advances, especially in the field of surgery, require better intraoperative anesthetic control and a minimum of adverse effects in the postoperative period. For this, new inducing agents such as propofol are currently available, anesthetic maintenance is achieved without the need for an inhalation agent. The main objective of this research was to present recent 
updates in the literature on total intravenous anesthesia, focused on its pharmacological effects and its main indications. A review of the literature and an analysis of publications on total intravenous anesthesia in the last 10 years was performed, using Pubmed and Scielo digital libraries, both articles in Spanish and English. Total intravenous anesthesia is the default solution for a patient at risk of malignant hyperthermia who requires general anesthesia, in addition to decreasing the risk of side effects of general anesthesia, such as postoperative nausea and vomiting, and avoiding contamination of ambient air with inhalation agents.

KEYWORDS: intravenous anesthesia, propofol, remifentanil, pharmacokinetics.

\section{INTRODUCCIÓN}

La provisión de anestesia general (AG) a través de agentes intravenosos (IV) solos, sin usar fármacos por vía inhalatoria (anestesia con gases), se conoce como anestesia intravenosa total (TIVA). Existe una justificación sólida razonable para el uso de la TIVA en algunos pacientes en los que es imposible o inconveniente la administración de anestésicos inhalados, o en situaciones en las que es impracticable el uso de los sistemas tradicionales de administración de anestésicos o no se dispone de ellos. En otros casos, el uso de TIVA podría hacer el proceso más eficiente y ventajoso para el paciente.

En comparación con la anestesia tradicional por inhalación, los beneficios inherentes de la TIVA a través de la infusión controlada por objetivo ( $\mathrm{TCl}$ la convierte en una técnica más efectiva y fácil de implementar por el anestesiólogo, mientras que al mismo tiempo ofrece una recuperación del paciente más rápido y cómoda. La TIVA es puramente una técnica anestésica $y$, cuando se usa en casos en los que se requiere tratar el dolor posoperatorio, se pueden aplicar estrategias multimodales de tratamiento del dolor como la administración de una anestesia regional antes de la TIVA para uso posoperatorio con el fin de acelerar la recuperación del paciente

En esta revisión se describe ella definición, indicaciones, ventajas y desventajas de la anestesia intravenosa total, basado en una recopilación sistemática de la literatura Latinoamericana y a nivel mundial, que espera sirva de apoyo científico para la comunidad médica. Se explorará las ventajas y desventajas de TIVA con un enfoque en los resultados de la anestesia de acuerdo al tipo de cirugía. Los problemas clínicos examinados incluirán la emergencia de la anestesia, náuseas y vómitos (NVPO), precondicionamiento isquémico y el trabajo emergente sobre el dolor postoperatorio agudo y crónico. 


\section{MATERIALES Y MÉTODOS}

Se realizó una revisión de la literatura y un análisis de publicaciones sobre los efectos, ventajas y desventajas de la anestesia intravenosa total publicados en los últimos 10 años, empleando bibliotecas digitales de Pubmed y Scielo, tanto de artículos en español e inglés. La búsqueda incluyó articulo desde enero del 2010 hasta diciembre del 2019, además se incluyeron artículos de mayor antigüedad que representen importancia histórica en la investigación. La búsqueda sistemática se realizó empleando términos del vocabulario MeSH: "intravenous anesthesia"OR "propofol" OR "remifentanil" OR "inhalation"; y del vocabulario DeCS: intravenous anesthesia, propofol, remifentanil, pharmacokinetics. Fueron seleccionados artículos de revisión de la literatura en idioma inglés y español. Se excluyeron artículos cuyo contenido abarcaba fisiopatología y tratamiento del cáncer diferenciado de tiroides. Se escogieron 20 artículos procedentes Pubmed y Scielo para el análisis documental.

\section{RESULTADOS}

Se define como anestesia total intravenosa o TIVA por sus siglas en inglés, a la combinación de varios agentes intravenosos para inducción y mantenimiento de la anestesia general, entre ellos: barbitúricos, hipnóticos no barbitúricos, opioides, agentes bloqueadores neuromusculares, $\alpha$ agonistas, anestésicos locales (lidocaína) entre otros, en ausencia absoluta de cualquier agente anestésico inhalado (1). La TIVA se ha vuelto más popular en los últimos 20 años debido a las propiedades farmacocinéticas y farmacodinámicas del propofol y la disponibilidad de opioides sintéticos de acción corta. El uso de TIVA tiene una serie de ventajas teóricas sobre los agentes inhalantes para mantener la AG. Los medicamentos utilizados para TIVA disminuyen el riesgo de efectos secundarios de $A G$, como náuseas y vómitos postoperatorios (NVPO) y evitan la contaminación del aire ambiental con los agentes inhalantes. A pesar de estas y otras ventajas potenciales, el uso de TIVA sigue siendo bajo. Existen inquietudes acerca de la mayor posibilidad de concienciación del paciente con TIVA en comparación con el uso de agentes inhalantes.

Indicaciones (2):

1. Técnica alternativa o coadyuvante a la inhalatoria.

2. Sedación en anestesia local o locorregional

3. Técnica de anestesia general para procedimientos quirúrgicos de alta o baja complejidad.

4. Anestesia fuera de las áreas quirúrgicas.

5. Reducción de la contaminación ambiental.

Las indicaciones específicas para TIVA se dan en la Tabla 1 . La TIVA es aplicable a casi todos los tipos de cirugía, pero tiene un valor particular en escenarios clínicos en los que se requiere una extubación despierta sin estrés y sin laringoespasmo. La TIVA confiere muchas ventajas sobre una técnica volátil convencional, particularmente un mejor perfil de recuperación con un menor riesgo 
de náuseas y vómitos postoperatorios, y puede facilitar el despertar intraoperatorio mientras se retiene la amnesia. El uso de TIVA para casos que requieren una secuencia de intubación rápida es controvertido, pero se practica con seguridad.

Tabla 1.

Indicaciones específicas para anestesia total intravenosa (TIVA)

Riesgo de hipertermia maligna
Síndrome de QT largo (QTc $\geq 500 \mathrm{~ms}$ )
Historia de NVPO severa
ENT 'sin cámara' y cirugía torácica
Pacientes con intubación / extubación difícil anticipada
Neurocirugía: para limitar el volumen intracraneal
Cirugía que requiere monitoreo neurofisiológico
Miastenia gravis / trastornos neuromusculares y situaciones en las
que los NMB son desventajosos
Anestesia en entornos no teatrales.
Transferencia de un paciente anestesiado entre entornos.
Cirugía Daycase
Docente en prácticas
Elección del paciente

\section{Drogas utilizadas pata TIVA}

En teoría, se puede usar cualquier combinación de hipnótico (s) intravenoso y opioide (s) y se describen técnicas sin opioides. En la práctica, la sinergia entre las infusiones de $\mathrm{TCl}$ de propofol y remifentanilo es altamente efectiva para obtener una respuesta contundente a los estímulos nocivos. Esta combinación de medicamentos logra el equilibrio entre la profundidad adecuada de la anestesia y la recuperación rápida. Los bolos intermitentes de agentes o las infusiones controladas manualmente pueden producir un efecto inadecuado.

\section{Propiedades de un agente anestésico ideal}

Hay varias propiedades en las que se puede pensar al considerar el agente anestésico ideal:

- Inicio y compensación rápidos.

- Recuperación rápida a la línea de base.

- Analgesia a concentraciones subanestésicas.

- efecto antiemético

- Mínima depresión cardiovascular y respiratoria.

- Ausencia de metabolitos activos.

- Metabolismo independiente del órgano. 
- Fácilmente titulable.

- No hay interacción con fármacos bloqueantes neuromusculares.

- Sin efectos tóxicos en otros órganos.

- Antioxidante, antiinflamatorio.

- Larga vida útil.

- Sin reacciones de hipersensibilidad o liberación de histamina.

- Seguro si se inyecta inadvertidamente en una arteria.

Ninguno de los agentes disponibles actualmente cumple con todos estos requisitos. Sin embargo, la TIVA con propofol tiene una serie de ventajas potenciales sobre los agentes inhalantes.

\section{Monitorización de la anestesia intravenosa total}

En la actualidad, las concentraciones plasmáticas de propofol no pueden medirse minuto a minuto de una manera práctica. Se puede lograr la calibración clínica del paciente individual antes del 'cuchillo a la piel' al observar los incrementos en las concentraciones en el sitio del efecto que muestran: Esta metodología proporciona tres puntos de calibración para guiar los objetivos mínimos y máximos que se utilizarán. Si se requiere parálisis neuromuscular, no debe administrarse hasta que se haya logrado una falta de respuesta al empuje de la mandíbula. La valoración de la concentración del sitio del efecto a la respuesta del paciente durante la cirugía es vital, particularmente porque la hipnosis excesiva es un problema más común que una profundidad inadecuada. Es posible utilizar el software farmacocinético de Tivatrainer para predecir las concentraciones necesarias del sitio del efecto en tiempo real durante la administración del fármaco. Este programa expone gráficamente las probabilidades del 50 y $95 \%$ de falta de respuesta a un evento nocivo (similar a 1 y 2 MAC para volátiles) cuando se usan simultáneamente $\mathrm{TCl}$ de propofol y remifentanilo.

\section{Recuperación de la anestesia}

La recuperación después de la anestesia y la cirugía es un proceso complejo que depende de las características del paciente, quirúrgico y anestésico, así como la presencia de cualquiera de las numerosas secuelas adversas.

La farmacocinética y farmacodinámica de las combinaciones de propofol-opioides en TIVA se han descrito con mayor detalle en los últimos 30 años. El propofol es muy adecuado para las técnicas de infusión continua, ya que su vida media sensible al contexto aumenta en solo 20 a 30 minutos con duraciones de infusión de 2 a 8 horas. El alto aclaramiento y la redistribución después de una infusión prolongada permiten un rápido retorno a la conciencia. La adición de un opioide a una técnica TIVA disminuye los requerimientos de propofol en aproximadamente un $50 \%$. Esto permite una recuperación aún más rápida después de la terminación del propofol y las infusiones de opioides. 
El tiempo de recuperación de la conciencia después de la anestesia con propofol-opioide depende predominantemente del opioide seleccionado y solo marginalmente de la duración de la infusión. El propofol-remifentanilo permite un retorno más rápido de la conciencia que el propofol en combinación con fentanilo, sufentanilo o alfentanilo. Clínicamente, se ha demostrado que el uso de TIVA mejora la recuperación en varios grupos y entornos de pacientes diferentes. EI TIVA basado en propofol se ha asociado con una recuperación mejorada y menores costos en comparación con sevoflurano para anestesia basada en consultorio. Esto ha resultado en una estancia más corta en la sala de recuperación, alta temprana y una mayor satisfacción del paciente. Sin embargo, la diferencia general es pequeña, con un tiempo total desde el final de la anestesia hasta el alta de 51 minutos en el grupo de propofol versus 62 minutos en el grupo de sevoflurano.

En neurocirugía, el tiempo hasta la extubación y la recuperación postoperatoria no fue diferente con la anestesia con propofol-remifentanilo TIVA que con la anestesia con sevoflurano-sufentanilo cuando ambos grupos fueron guiados por un protocolo de índice biespectral (BIS). Los autores teorizaron que el uso de monitoreo BIS en ambos brazos del estudio podrían haber mitigado las ventajas farmacodinámicas de TIVA. Un estudio anterior encontró una recuperación más rápida del sevoflurano que de la TIVA durante la cirugía de la columna vertebral cuando la administración anestésica fue guiada por potenciales evocados somatosensoriales. Sin embargo, también se ha demostrado que la monitorización de la profundidad de la anestesia permite una mejor recuperación y una disminución del uso de propofol durante la TIVA.

\section{Náuseas y vómitos postoperatorios (NVPO)}

La NVPO con frecuencia complica la cirugía y la anestesia. las encuestas de pacientes indican constantemente que es una de las experiencias más desagradables en el período perioperatorio. A pesar de los avances significativos en nuestro conocimiento de NVPO y la introducción de nuevos medicamentos antieméticos, la incidencia general de NVPO se estima en alrededor del $30 \%$. En grupos de alto riesgo, esta incidencia es tan alta como $80 \%$. Los pacientes informan que evitar la NVPO es una preocupación mayor que evitar el dolor postoperatorio y expresan su disposición a pagar hasta $\$ 100$ de su bolsillo por un antiemético eficaz. La NVPO puede causar tiempos de recuperación prolongados y una mayor atención de enfermería para todos los procedimientos, así como un ingreso inesperado después de una cirugía ambulatoria. Todos estos factores aumentan los costos médicos generales.

La TIVA con propofol se asocia con una menor incidencia de NVPO en comparación con los agentes inhalantes. El uso de TIVA reduce el riesgo de NVPO en aproximadamente un $25 \%$. El efecto antiemético del propofol es más pronunciado en el postoperatorio temprano, con un número necesario para tratar (NNT) = 5 para disminuir la aparición de NVPO en las primeras 6 horas. El propofol, utilizado como parte de TIVA, es efectivo en todos los pacientes para reducir el riesgo basal de NVPO. 
El propofol subhipnótico también ha demostrado ser más eficaz que el placebo para el tratamiento de NVPO. La concentración media de propofol asociada con una reducción del $50 \%$ en las náuseas es de $343 \mathrm{ng} / \mathrm{ml}$. Esto se puede lograr con un bolo de $10 \mathrm{mg}$ de propofol seguido de una infusión de $10 \mu \mathrm{g} / \mathrm{kg} / \mathrm{min}$. Alternativamente, se ha demostrado que los bolos de $20 \mathrm{mg}$ de propofol administrados a través de un dispositivo controlado por el paciente en la unidad de cuidados postanestésicos reducen la NVPO y permiten el alta temprana. Aunque el mecanismo exacto de acción del propofol en la reducción de NVPO no se ha dilucidado, se han propuesto varios mecanismos, incluido un efecto depresor directo en la zona de activación de quimiorreceptores, núcleos vagales y otros centros implicados en NVPO. En modelos animales, se ha demostrado que el propofol disminuye la transmisión nerviosa sináptica en la corteza olfatoria y disminuye los niveles de serotonina en el área postrema.

\section{Protección miocárdica}

Se ha demostrado que los agentes anestésicos volátiles ofrecen un efecto cardioprotector debido al preacondicionamiento isquémico durante la cirugía de revascularización coronaria. El efecto cardioprotector relativo del propofol es controvertido. Se ha informado que el propofol mejora la capacidad antioxidante de los eritrocitos y los tejidos y, por lo tanto, proporciona protección dependiente de la dosis durante la isquemia y la reperfusión. En modelos animales se ha demostrado que el propofol produce un efecto cardioprotector de hasta 48 horas.

\section{Dolor postoperatorio}

La mayoría de los pacientes experimentan dolor postoperatorio, que se asocia con resultados clínicos y económicos adversos. Un estudio reciente sugirió que alrededor del $86 \%$ de los pacientes experimentaron dolor después de la cirugía; De estos, el 75\% tenía dolor moderado o extremo durante el período posquirúrgico inmediato, y el $74 \%$ todavía experimenta estos niveles de dolor después del alta. Hay alguna evidencia emergente de que el tipo de anestésico podría afectar el nivel de dolor postoperatorio. Los modelos animales han demostrado que los anestésicos volátiles causan hiperalgesia al salir de la anestesia (posiblemente debido a la inhibición de los receptores nicotínicos de acetilcolina en el cerebro y la médula espinal) y que el propofol puede tener un efecto antinociceptivo periférico.

Cirugía Sinusal

La TIVA ha demostrado en algunos estudios que impacta la pérdida de sangre durante la cirugía endoscópica de seno, con los consiguientes efectos sobre la estabilidad cardiovascular y el campo visual durante la cirugía.

Cirugía de quemaduras

La TIVA basada en ketamina se usa con frecuencia para operaciones importantes que requieren anestesia general en pacientes con quemaduras graves, particularmente si hay una lesión por inhalación concomitante. Un estudio reciente descubrió que el uso de ketamina, fentanilo y propofol en este entorno era seguro y daba como resultado menos requisitos de presión (con 
estabilidad cardiovascular equivalente durante los casos de TIVA) que la anestesia por inhalación.

Función pulmonar postoperatoria

Tiefenthaler et al realizaron recientemente el primer estudio que investigaba la influencia de la técnica anestésica en la función pulmonar postoperatoria. El estudio se realizó en 60 pacientes sometidos a cirugía de disco lumbar en posición prono. Al igual que en otros estudios previos sobre la función pulmonar postoperatoria, los investigadores encontraron que los parámetros de la función pulmonar disminuyeron después de la cirugía, independientemente del tipo de anestesia administrada. Sin embargo, la disminución de la capacidad residual funcional fue marginalmente mayor con TIVA que con la anestesia inhalatoria.

\section{DISCUSIÓN}

Larsen et al compararon la recuperación de la función cognitiva después de propofolremifentanil TIVA con la recuperación después de la anestesia con desflurano y sevoflurano. El grupo TIVA exhibió una emergencia significativamente más rápida que aquellos que recibieron desflurano o sevoflurano, sin diferencias entre los agentes inhalantes. El retorno de la función cognitiva medido por la prueba de puntos de Trieger y la prueba de sustitución de símbolos de dígitos fue significativamente más rápido con TIVA que con desflurano y sevoflurano hasta 60 minutos después de la administración de la anestesia. No hubo diferencias significativas entre los grupos a los 90 minutos.

La TIVA guiada por BIS también puede disminuir el riesgo de conciencia en comparación con la TIVA de rutina. Un gran estudio examinó las características de recuperación de 1.158 pacientes sometidos a cirugía de casos mixtos. Los pacientes fueron asignados al azar a inducción y mantenimiento de propofol (TIVA), inducción de propofol e isoflurano/N2O o sevoflurano/N2O, o inducción y mantenimiento de sevoflurano por inhalación. No se utilizó la monitorización de la profundidad de la anestesia. Hubo menos NVPO con TIVA, pero no hubo diferencia en el tiempo hasta el estado mental al despertar, el tiempo de recuperación, el tiempo de alta o los ingresos hospitalarios no planificados entre los grupos.

Un estudio reciente demostró que la TIVA libre de opioides con una combinación de propofol, ketamina y dexmedetomidina fue capaz de reducir el riesgo absoluto de desarrollar NVPO en un 17,3\% (NNT = 6) en comparación con la anestesia inhalatoria con opioides. De particular interés fue el hecho de que ambos grupos recibieron triple profilaxis con NVPO con un parche transdérmico de escopolamina, dexametasona y ondansetrón. El efecto de la TIVA libre de opioides fue, por lo tanto, adicional a la mejor práctica de terapia antiemética.

Una revisión sistemática de 58 estudios también mostró que la TIVA con propofol es más efectiva que la anestesia inhalatoria para reducir las náuseas y los vómitos posteriores al alta (NVPOA). Las NVPOA se reconocen cada vez más como un problema importante, con una incidencia reportada del $37 \%$ en las primeras 48 horas después del alta de la cirugía 
ambulatoria y pueden ser difícil de tratar, ya que los pacientes ya no pueden recibir agentes antieméticos IV. Se recomienda el uso de TIVA como parte de un enfoque multimodal para todos los pacientes con alto riesgo de NVPO o NVPOA.

El efecto cardioprotector relativo del propofol es controvertido: un metaanálisis de 22 estudios mostró una tasa significativamente menor de infarto de miocardio y muerte en pacientes sometidos a cirugía cardíaca con desflurano o sevoflurano en comparación con TIVA. Un estudio retrospectivo de 10.535 pacientes sometidos a cirugía cardíaca concluyó que el sevoflurano y el propofol ofrecen algunas, pero diferentes, propiedades cardioprotectoras.

Los resultados de los ensayos controlados aleatorios (ECA) son contradictorios. Algunos ECA han concluido que la TIVA no parece ofrecer ninguna protección miocárdica en pacientes sometidos a cirugía cardíaca, en comparación con los agentes volátiles, mientras que otros no han encontrado diferencias cuando se usó cualquiera de las técnicas. Es importante señalar que todos estos estudios utilizaron troponina postoperatoria que aumenta como un marcador de necrosis miocárdica. La relevancia clínica de esto es incierta. De hecho, puede ser muy difícil, si no virtualmente imposible, extrapolar reducciones pequeñas, pero estadísticamente significativas en los marcadores bioquímicos de necrosis miocárdica observados con anestésicos volátiles demostrables en el resultado.

El efecto antinociceptivo periférico de propofol es controversial, pero Cheng et al compararon el propofol con el isoflurano en 80 mujeres sometidas a cirugía uterina. La variable de resultado primaria fue el dolor informado en una escala analógica visual. Encontraron que la TIVA con propofol resultó en un uso significativamente menor de dolor y morfina en las primeras 24 horas ( $p<0.01$ para ambos resultados). Por el contrario, en la cirugía del oído medio, Mukherjee et al encontraron que los pacientes que recibieron TIVA experimentaron más dolor postoperatorio en la sala de recuperación y requirieron más morfina. Lo más interesante es que un ECA grande reciente $(n=366)$ que evalúa los efectos de la anestesia en el síndrome de dolor crónico postoracotomía (SDCPT) encontró una prevalencia significativamente menor de SDCPT en pacientes que reciben TIVA con propofol-remifentanilo que en aquellos que reciben anestesia inhalatoria.

El grupo TIVA tuvo menos alodinia y SDCPT a los 3 meses $(38,2 \%$ frente a 56,5\%, $p=0,001)$ y a los 6 meses (33,5\% frente a 50,6\%, p =0,002). Los autores teorizaron que esta reducción podría deberse a los siguientes factores: El efecto antinociceptivo periférico del propofol, efectos antioxidantes del propofol, efecto neuroprotector del propofol en los nervios intercostales lesionados y la inhibición del subtipo NMDA (N-metil-D-aspartato) del receptor de glutamato por el propofol. Además, se ha demostrado que propofol TIVA reduce la hiperalgesia inducida por remifentanilo.

En un estudio realizado por Shin et al de pacientes sometidas a cirugía de cáncer de mama, el grupo que recibió propofol durante la anestesia basada en remifentanilo en dosis altas informó una mejor analgesia postoperatoria con un consumo de morfina significativamente menor a 
las 24 horas que el grupo de sevoflurano. El receptor NMDA está involucrado en la génesis de la hiperalgesia, por lo que este efecto también puede estar relacionado con el antagonismo del receptor NMDA.

Estudios han documentado que la anestesia intravenosa total influye positivamente sobre el sangrado en cirugía de senos paranasales. Sin embargo, un metaanálisis reciente de 42 estudios no encontró diferencias en la pérdida de sangre, la frecuencia cardíaca o la presión arterial entre la TIVA y la anestesia por inhalación. Solo 7 estudios informaron una puntuación de visibilidad, pero favorecieron al grupo TIVA $(p<0,001)$.

\section{CONCLUSIONES}

La anestesia total intravenosa es la solución predeterminada para un paciente con riesgo de hipertermia maligna que requiere anestesia general. Es probable que la mala educación y capacitación en el uso de esta técnica genere un riesgo significativo de conciencia. El uso de propofol y remifentanilo por infusión controlada por objetivo y el cumplimiento de recomendaciones simples evitarán la mayor parte de este riesgo.

\section{REFERENCIAS}

1. Ramírez H. Anestesia total intravenosa. Rev Mex Anestesiol. Vol. 38 (3): pp S430-S432. 2015.

2. Abad A. Introducción a la anestesia total intravenosa. Capítulo 1: Aspecto práctivo de TIVA. Disponible https://files.sld.cu/anestesiologiacardiovascular/files/2011/06/capitol01.pdf; 2011.

3. Miller T. Total intravenous anesthesia and anesthetic outcomes. J Cardiothorac Vasc Anesth. Vol. 29 Suppl 1:S11-S15. doi:10.1053/j.jvca.2015.01.022. 2015.

4. Anderson B. Practicalities of Total Intravenous Anesthesia and Target-controlled Infusion in Children. Anesthesiology. Vol.131(1):164-185. doi:10.1097/ALN.0000000000002657. 2019.

5. Goh A. A follow-up survey of total intravenous anesthesia usage in children in the U.K. and Ireland. Paediatr Anaesth. Vol. 29(2):180-185. doi:10.1111/pan.13556. 2019.

6. Zuleta A. Total intravenous anaesthesia versus inhaled anaesthetics in neurosurgery. Rev Colomb Neurocir. Vol 43 (1):9-14. 2015.

7. Tafur L. Anestesia total intravenosa: de la farmacéutica a la farmacocinética. Rev Col Anest. Vol. 38 (2): 215-231. 2010.

8. Navarrete V. Anestesia Total Intravenosa (TIVA) - 2018. Rev Cub Anest Reanim. Vol. 18(1): e531. 2019. 
9. Z Al-Rifai M. Principles of total intravenous anaesthesia: practical aspects of using total intravenous anaesthesia. BJA Education. Vol. 16 (8): 276-280. doi: 10.1093/bjaed/mkv074. 2016.

10. Nimmo A. 5th National Audit Project (NAP5). Accidental Awareness during General Anaesthesia in the United Kingdom and Ireland Report and Findings-Chapter 18. Total intravenous anaesthesia. The Royal College of Anaesthetists. 2014.

11. Kocaturk $O$. Recovery characteristics of total intravenous anesthesia with propofol versus sevoflurane anesthesia: a prospective randomized clinical trial. J Pain Res. Vol. 11:12891295. doi:10.2147/JPR.S164106. 2018.

12. Wong $\mathrm{S}$. The analgesic effects of intraoperative total intravenous anesthesia (TIVA) with propofol versus sevoflurane after colorectal surgery. Medicine (Baltimore). Vol. 97(31):e11615. doi:10.1097/MD.0000000000011615. 2018.

13. Urman R. Anestesia de bolsillo (2a edición ed). Capitulo 10: Anestesdia intravenosa total. Lippincott-Wolters Kluwer. 2014.

14. Miller R. Miller Anestesia. Cap. 13: Anestesia intravenosa total. (Séptima edición ed., Vol. 1). España: Elsevier-Saunders. 2015. 